\title{
Commentary: No filter-The real prognosis of kidney injury after ventricular assist device implantation
}

Jason J. Han, MD, and Pavan Atluri, MD

\footnotetext{
From the Division of Cardiovascular Surgery, Department of Surgery, University of Pennsylvania, Philadelphia, $\mathrm{Pa}$.

Disclosures: Authors have nothing to disclose with regard to commercial support.

Received for publication April 10, 2019; accepted for publication April 10, 2019; available ahead of print May 21 2019.

Address for reprints: Pavan Atluri, MD, Division of Cardiovascular Surgery, Hospital of the University of Pennsylvania, 3400 Spruce St, 6 Silverstein Pavilion, Philadelphia, PA (E-mail: Pavan.Atluri@uphs.upenn.edu). J Thorac Cardiovasc Surg 2020;159:487-8

$0022-5223 / \$ 36.00$

Copyright (c) 2019 by The American Association for Thoracic Surgery

https://doi.org/10.1016/j.jtcvs.2019.04.042
}

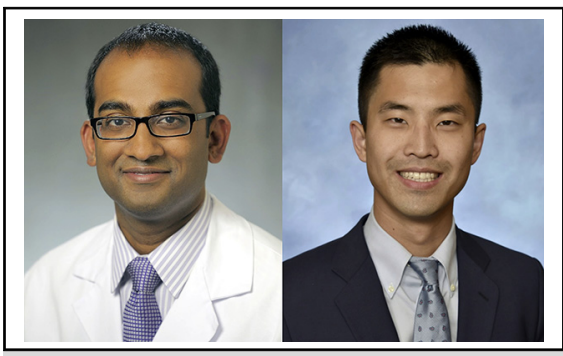

Pavan Atluri, MD (left), and Jason J. Han, MD (right)

Central Message

Acute kidney injury is one of the most common complications after ventricular assist device implantation and has significant prognostic implications.

See Article page 477. insults secondary to poor perfusion or congestion, the true scope of AKI's clinical impact is only beginning to be fully appreciated. ${ }^{5,6}$ AKI is independently associated with increased mortality. ${ }^{1}$ In previous studies, as many as a third of all patients have been shown to require at least temporary renal replacement therapy (AKI-D), which significantly worsens prognosis, limits quality of life, and contraindicates heart transplantation. ${ }^{7}$ As the prevalence of LVAD implantation, particularly for destination therapy in populations at higher risk, continues to increase in this country, the national burden of this complication, prognostically and financially, will only grow more significant.

It is at this critical juncture that the study by Silver and colleagues $^{8}$ in this issue of the Journal contributes several major insights to the literature. By examining the National Inpatient Sample (NIS), which is the largest all-payer inpatient database in the United States, they analyzed more than 8000 admissions from 2008 to 2013 across various regions and types of practices. They noted that more than half of these cases were complicated by AKI, and $10 \%$ of the cases of AKI were severe enough to require dialysis. The in-hospital mortality was 3 times higher for patients with AKI and more than 10 times higher for those with AKI-D. None of these patients who required renal replacement therapy were eligible for heart transplantation, and only $30 \%$ were able to return home. Unsurprisingly, risk factors that predisposed to AKI in this study were markers of a critically ill state, such as shock, ventilator dependence, existing renal dysfunction, and emergency need for mechanical circulatory support.

What was surprising, however, was just how many of these patients in this national database selected for LVAD implantation were critically ill, approximately $40 \%$ with chronic kidney disease and 30\% requiring preoperative mechanical ventilation. These findings encourage us to consider the fact that such high rates of AKI and AKI-D in this study may reflect, in addition to the invasive nature of the intervention, a need nationally for more stringent patient selection, indicating that although LVADs provide lifesaving therapy for most beneficiaries, implantation should be carefully considered in patients who are actively dying with signs of global deterioration. Perhaps for this reason, despite a notable increase in volume and experience nationally through the study's duration, the study noted an increase in rates of AKI.

Ultimately, Silver and colleagues ${ }^{1}$ deserve our congratulations for this important and timely work. This is the largest study to date on this subject and highlights the scale of the burden that AKI and AKI-D after LVAD implantation poses nationally. Especially with respect to AKI-D, this study provides prognostic implications that can guide patient and family discussions, both preoperatively and postoperatively. As the volume of LVAD implantation will surely only continue to increase in the future, these considerations are imperative to ensure that patient outcomes, including quality of life, will also continue to improve. 


\section{References}

1. Muslem R, Caliskan K, Akin S, Sharma K, Gilotra NA, Constantinescu AA, et al. Acute kidney injury and 1-year mortality after left ventricular assist device implantation. J Heart Lung Transplant. 2018;37:116-23.

2. Anjum A, Kurihara C, Critsinelis A, Kawabori M, Sugiura T, Civitello AB, et al. Acute kidney injury after implantation of a left ventricular assist device: a comparison of axial-flow (HeartMate II) and centrifugal-flow (HeartWare HVAD) devices. J Artif Organs. 2018;21:285-92.

3. Harmon DM, Tecson KM, Lima B, Collier JD, Shaikh AF, Still S, et al. Outcomes of moderate-to-severe acute kidney injury following left ventricular assist device implantation. Cardiorenal Med. 2019;9:100-7.

4. Kilic A, Chen CW, Gaffey AC, Wald JW, Acker MA, Atluri P. Preoperative renal dysfunction does not affect outcomes of left ventricular assist device implantation. J Thorac Cardiovasc Surg. 2018;156:1093-101.e1.
5. Hasin T, Topilsky Y, Schirger JA, Li Z, Zhao Y, Boilson BA, et al. Changes in renal function after implantation of continuous-flow left ventricular assist devices. J Am Coll Cardiol. 2012;59:26-36.

6. Kirklin JK, Naftel DC, Kormos RL, Pagani FD, Myers SL, Stevenson LW, et al. Quantifying the effect of cardiorenal syndrome on mortality after left ventricular assist device implant. J Heart Lung Transplant. 2013;32: 1205-13.

7. Naik A, Akhter SA, Fedson S, Jeevanandam V, Rich JD, Koyner JL. Acute kidney injury and mortality following ventricular assist device implantation. Am J Nephrol. 2014;39:195-203.

8. Silver SA, Long J, Zheng Y, Goldstone AB, Franz D, Chang TI, et al. Outcomes after left ventricular assist device implantation in patients with acute kidney injury. J Thorac Cardiovasc Surg. 2020;159: 477-86.e3. 